\title{
Turning Science into Song: How It All Began ${ }^{1}$
}

\author{
Stephen Baird, $\mathbf{M D}^{2}$
}

How It All Began is a modern version of the first chapter of Genesis. It may be sung to "Hark! The Herald Angels Sing", the popular $18^{\text {th }}$ century Christmas carol. The right hand column may be considered explanatory side notes.

Bang! It went, and off we go, fourteen billion years ago. First the fireball, hot and dense-the energy within, immense. Then the Higgs Field yielded matter; particles began to scatter; and were promptly blown to bits when they met their opposites. This is most of what we know of fourteen billion years ago.
Modern theory postulates a beginning of the cosmos from a "singularity," an infinitely small, hot, and dense point that is undefinable by the current laws of physics (Figure 1). The Big Bang exploded from this undefined situation. The Higgs Field is named for one of six physicists who postulated a mechanism for converting the energy of the Big Bang into matter in 1964. This was confirmed in 2013. The particles that emerged from the energy field always emerge in pairs, a so-called particle and anti-particle. These totally annihilate when they come in contact.

\footnotetext{
${ }^{1}$ Received on March 15, 2016. Accepted on March 18, 2016. Last revisions received on May 20, 2016.

${ }^{2}$ MD (Doctor of Medicine). Professor Emeritus, University of California, San Diego, California, USA. E-mail: stephen.baird3@gmail.com. As for other contributions published in Life: The Excitement of Biology, the copyright holder is(are) the author(s) of the contribution, in this case, Doctor Stephen Baird. Figure 1 was produced by NASA and is not copyrighted by the author.
} 
Though it's harder to create when particles annihilate, they released such energy that all four forces were set free. Then the heat and radiation pumped up history's first inflation. And, as our new cosmos grew, bits of matter made it through. That's what our equations show microseconds from the go.

Matter started filling space when particles flew every place. Most of them were just debris so atoms formed from only three. Nuclei were made of protons, often clumped along with neutrons-and electron clouds were bound in discrete shells, buzzing 'round. Atoms popped out from the glow of the Big Bang, long ago.
Some matter: protons, neutrons, etc., survived but we're not sure why we are made of matter, not anti-matter. The four fundamental forces that emerged after the Big Bang were: the strong nuclear force, the weak nuclear force, electromagnetism, and gravitation. Alan Guth proposed that there was a brief period of hyperinflation when the cosmos actually expanded faster than the speed of light. This does not violate Einstein's concept that nothing in the universe can go faster than the speed of light. The whole universe, very small at that time, expanded faster than the speed of light.

In the very hot, early universe, myriad particles, stable and unstable, precipitated from the expanding, cooling energy field. Protons, neutrons, and electrons are particularly stable compared to the others. For example, the Higgs Boson, the postulated first mass, rapidly decays to other particles.

The standard model of the atom: a nucleus of protons and neutrons with electrons orbiting around it like planets around the sun is better conceived as a nucleus with clouds of electrons around it. Quantum physics and probability describe the likely location of any given electron particle at any given time. 
Eons later things had cooled; the universe had been retooled. Hydrogen and helium were building blocks for things to come. Then the force of gravitation led to even more creation.

Fusion made the first stars shine and more baryons combine. That's what our equations show of thirteen billion years ago.

Populations came and went, for when their hydrogen was spent, little stars collapsed and died. Bigger ones were glorified. With a flash, these stars were shattered; and new elements were scattered. Then these clouds of starry dust fused again with cosmic lust. That's what Hubble's pictures show about ten billion years ago.
Ralph Alpher, a student of George Gamow, calculated in 1948 that both hydrogen and helium and a tiny bit of lithium could form from the Big Bang. The synthesis of all the other elements required fusion in stars. For this to happen, the universe needed to cool enough so that the relatively weak force of gravity could collapse these early clouds of hydrogen and helium into stars where compression due to their enormous mass would eventually ignite fusion reactions. Stars can be thought of as hydrogen bombs where the explosive pressure of the ongoing fusion reactions is balanced by the compressive force of gravity, producing a huge sphere synthesizing heavier and heavier elements.

We divide stars into populations according to their content of heavier elements. When stars (novas) explode they scatter the elements they have synthesized through space where they can mix with primeval hydrogen and helium, collapse again and form a new star. Our sun is probably a second or third generation star because of its relatively high content of heavier atoms, determined by spectroscopy.

When the Hubble telescope looks into deep space, it is looking back in time. What we see ten billion light years away happened ten billion years ago. 
Planets formed from dust and gas, sized according to their mass.

They accrete by gravity

(Accrete means forming gradually.)

Back when Earth was somewhat hotter life could start in pools of water;

RNA could replicate,

then took protein for a mate.

That's what modern studies show about four billion years ago.

Then we think that RNA, through its mutant, DNA, rearranged to code for genes and translate them to proteins. Most genes spelled out protein enzymes that replaced old ribozymes. Those producing lipids well found themselves inside a cell. That's what micro-fossils show From three billion years ago.
The new elements from exploding stars can form interstellar dust and gas. When gravity collapses such clouds, a star tends to form at the center and smaller aggregates, planets such as Earth or Jupiter, may accrete in orbits around the new star.

When a planet orbits in the "Goldilocks Zone" where water can be liquid, the organic chemistry necessary for life is possible. Pools such as in Yellowstone or hydrothermal vents in the ocean are both postulated to have been environments where life could have started. Stanley Miller and Harold Urey first proposed the relevant chemistry in 1953. Since then, many others have contributed to our understanding of how life may have spontaneously arisen.

Students of the origin of life think that RNA preceded DNA. We are unsure of the exact sequence of events that led to the canonical DNA to RNA to protein pathway of life today. But experiments have shown plausible pathways for the abiotic synthesis of amino acids, lipids, carbohydrates, and nucleic acids, the four most important families of the molecules of life. Marrying them together into a cooperative cell is still poorly understood although we have made tremendous progress in the last 60 years.

We also know that some RNA molecules (ribozymes) could and still do function as enzymes. They probably preceded protein enzymes. 
Living things were first marine; early cells were bluish-green. Microbes formed stromatolites spreading out on seashore sites. Then somewhere some more mutation, followed by more incubation, made some creatures exoshelled, leaving fossils where they dwelled. This is what the data show-six hundred million years ago.

Simple creatures in the sea increased in their diversity. Sponges, plants, and fish evolved. Soon the shore was reinvolved. Amphibians crawled from the water, ate each other--what a slaughter! Big ones were selected for and produced the dinosaur. That's what tons of fossils show Two hundred million years ago.
We think that life started in water and gradually became more complex. At first the atmosphere had no elemental oxygen. Then blue-green bacteria began to produce oxygen as a byproduct of their photosynthetic metabolism. This made other organisms (of which we are the descendants) possible. Stromatolites of blue-green bacterial fossils are still present on the west coast of Australia.

As diversity increased, animals with protective armor, shells, began to arise about 540 million years ago. We call this sudden increase in the variety of fossils the Cambrian Explosion. We should note however that this "explosion" happened over tens of millions of years. Stephen J. Gould describes it in his book, "Wonderful Life."

Species were not stable as Darwin and Wallace recognized in their joint letter to the Linnean Society in London in 1858 . Diversity increased and tetrapods left the water. Fish fins modified gradually into limbs suitable for walking on the shore as is illustrated by the fossils of Tiktaalik roseae, Eusthenopteron, and Acanthostega in the exhibition at the American Museum of Natural History in New York City. This occurred over tens of millions of years in the Devonian Period, about 420-360 million years ago. Swim bladders and lungs also developed during this time.

The dinosaurs arose later, in the Triassic Period, around 230 million years ago. 
Then there came an asteroid-Bang! and life forms were destroyed. Smoke and dust flew through the sky and all the plants began to die. This disastrous complication caused the dinosaur's starvation. Furry animals survived. It's from these that we're derived. That's what still more fossils show-Some sixty million years ago.

Selection then could recommence. The data grow. They're making sense. In Africa, the apes diverged. Australopithecines emerged. Lucy got stuck in the mire. Homo habilis made fire. Copies of each skeleton pose in the Smithsonian. That's what lots more fossils show from three million years ago.
The asteroid strike referred to here is the Chicxulub event in the Yucatán, México, about 65 million years ago. There were five major die-offs documented in the fossil record. The Chicxulub event is the most recent. Catastrophic volcanic activity has also been implicated as a cause of these major die-offs.

Ancestors of mammals were already present and their ability to control their body temperature may have given them a survival advantage so that their descendants came to dominate the land animals. Note that all the dinosaurs did not die at once. It probably took a million years or more before they were gone and only represented today by birds.

As diversity kept increasing, hominids began to appear. The molecular and fossil evidence indicates that the ancestors of apes and humans separated about six to seven million years ago. The exact sequence of descent to humans is a subject of intense study and debate. It is probably better viewed as a bush than a linear path. And, we think that the branches of the bush probably occasionally interbred, sometimes in friendly, sometimes in not so friendly fashion. The famous skeleton, "Lucy" is about 3.2 million years old. Homo habilis, "handy man," lived from about 2.8 to 1.5 million years ago. Our ancestors were starting to make and use tools. They now stood upright but still had Chimpanzee-sized brains. 
In another million years

$H$. erectus then appears-crude tool makers, bigger brains and coming inside when it rains. Genes mutating, changing species, folks migrating, spreading feces, showing where they came and went, what they ate, how time was spent. They were always on the go about a million years ago.

That's all nice, but that's not all; next appeared Neanderthal. His descendants are at play throughout the NFL today. Then Cro-Magnon made his entrance; others faded from existence.

Bronze age man began to write, so you can read the rest tonight. This is How It All Began-from the Big Bang, up to man.
Homo erectus, as his name implies, stood upright, was a tool user, and had a much larger brain than previous Homo species. We give him credit for more intelligence. H. erectus left Africa and migrated into Eurasia where we think he was ancestral to Neanderthal. Those staying behind in Africa eventually were ancestral to Homo sapiens by about two hundred thousand years ago. Coprolite, fossilized feces, has been identified from Neanderthals and dated to about 50,000 years ago. It provides evidence of what these people ate.

Coprolite with human DNA of much more recent origin, 14,300 years old, has been found in the Paisley caves in Oregon. This is regarded as evidence of very early migration of humans into North America.

Homo neanderthalensis, named after a valley in Germany where the first cranium was found, had a brain a little larger on average than ours. He was a tool maker but went extinct about 25,000 years ago, before the Neolithic Age. Thanks to the work of Svante Paabo, we know that Neanderthal DNA is about $99.5 \%$ the same as ours. We also have good evidence that Europeans and Asians usually carry about 2-4\% genes of Neanderthal origin in their DNA. So they interbred with our Cro Magnon ancestors. Writing began in Sumer about 3,000 BCE. We have lots of their clay tablets and have been able to read their creation stories as well as laws and business records. Our Biblical creation stories clearly contain elements of these earliest, written records. 


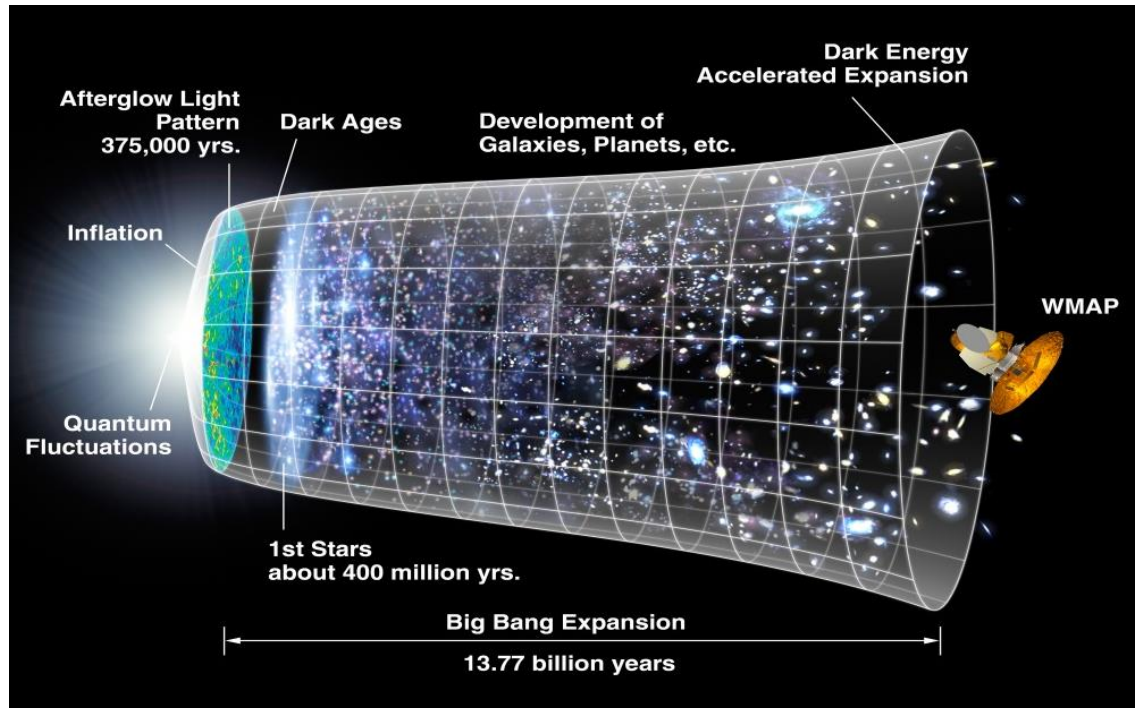

Figure 1. The Big Bang and its expansion, at the universal scale. Image taken from http://map.gsfc.nasa.gov/media/060915/index.html 